\title{
CONTINUITY OF THE DENSITY OF A GAS FLOW IN A POROUS MEDIUM ${ }^{1}$
}

BY

\author{
LUIS A. CAFFARELLI AND AVNER FRIEDMAN
}

\begin{abstract}
The equation of gas in a porous medium is a degenerate nonlinear parabolic equation. It is known that a unique generalized solution exists. In this paper it is proved that the generalized solution is continuous.
\end{abstract}

0. Introduction. The density $u(x, t)$ of gas in a porous medium satisfies the equation

$$
\partial u / \partial t=\Delta u^{m} \quad(m>1)
$$

for $x \in R^{n}, t>0$, and an initial condition

$$
u(x, 0)=u_{0}(x) .
$$

Here $u_{0}(x)>0$ and $u(x, t)>0$. The equation $(0.1)$ is a nonlinear parabolic equation, degenerating at the points where $u=0$. The concept of a solution of $(0.1),(0.2)$ is taken in some weak sense (to be defined precisely in $\S 1)$. The purpose of this paper is to prove that

$$
u(x, t) \text { is continuous. }
$$

This result is known for $n=1$; see [9], [10], [1] and [5].

In $\$ 1$ we state this result more precisely, giving also a uniform modulus of continuity. In $\$ \S 2$ and 3 we establish preliminary estimates. The proof of $(0.3)$ for $t>0$ is given in $\$ 4$ and, for $t=0$, in $\$ 5$.

1. The main results. Let $u_{0}(x)$ be a function defined in $R^{n}$ and satisfying:

$$
\begin{gathered}
0 \leqslant u_{0}(x) \leqslant N \quad(N<\infty), \\
\int_{R^{n}}\left(u_{0}(x)\right)^{2} d x<\infty,
\end{gathered}
$$

$u_{0}(x)$ is continuous in $R^{n}$, and uniformly Hölder continuous in every compact set where $u_{0}>0$.

Received by the editors August 7, 1978.

AMS (MOS) subject classifications (1970). Primary 35K55.

${ }^{1}$ This work is partially supported by National Science Foundation Grants 7406375 A01 and MC 575-21416 A01. 0002-9947/79/0000-0353/\$04.75 
We consider the Cauchy problem

$$
\begin{gathered}
\partial u / \partial t=\Delta u^{m} \quad \text { in } R^{n} \times(0, \infty), \\
u(x, 0)=u_{0}(x) \quad \text { in } R^{n},
\end{gathered}
$$

where $m$ is a fixed number, $m>1$.

By a solution of (1.4), (1.5) we mean a function $u(x, t)$ such that, for any $T<\infty$,

$$
\int_{0}^{T} \int_{R^{n}}\left[(u(x, t))^{2}+\left|\nabla_{x} u^{m}(x, t)\right|^{2}\right] d x d t<\infty
$$

and

$$
\int_{0}^{T} \int_{R^{n}}\left(u \frac{\partial f}{\partial t}-\nabla_{x} u^{m} \cdot \nabla_{x} f\right) d x d t+\int_{R^{n}} u_{0}(x) f(x) d x=0
$$

for any continuously differentiable function $f$ with compact support in $R^{n} \times[0, T)$.

We recall [11] that under the conditions (1.1), (1.2), there exists a unique solution.

Other concepts of a solution can be given which allow for a different decay condition at $x=\infty$ than in (1.2). The results of this paper are not affected by working with these other concepts of a solution.

The solution $u(x, t)$ can be obtained as a limit of solutions $u_{\eta}(x, t)(\eta \downarrow 0)$ of the equation (1.4) with the initial condition

$$
u(x, 0)=u_{0}(x)+\eta \quad \text { in } R^{n}
$$

see [11]. Notice that the solution $u_{\eta}$ of (1.4), (1.8) is taken in the classical sense, $u_{\eta}<u_{\eta^{\prime}}$ if $\eta \leqslant \eta^{\prime}$, and

$$
\eta<u_{\eta}(x, t) \leqslant N+\eta \quad \text { in } R^{n} \times(0, \infty) .
$$

We define the parabolic distance between two points $\left(x^{1}, t^{1}\right),\left(x^{2}, t^{2}\right)$ by

$$
d\left(\left(x^{1}, t^{1}\right),\left(x^{2}, t^{2}\right)\right)=\left|x^{1}-x^{2}\right|+\left|t^{1}-t^{2}\right|^{1 / 2} .
$$

When we shall speak of a modulus of continuity of a function $v(x, t)$, we shall always mean the distance between two points to be the parabolic distance.

We now introduce two moduli of continuity:

$$
\begin{aligned}
\omega_{e}(r) & =C|\log r|^{-\varepsilon} \quad(0<\varepsilon<2 / n), \\
\tilde{\omega}(r) & =C 2^{-c|\log r|^{1 / 2}}
\end{aligned}
$$

where $C>0, c>0$.

The main result of the paper is stated in the following theorem.

THEOREM 1.1 (i) The solution $u$ of (1.4), (1.5) is continuous in $R^{n} \times[0, \infty)$;

(ii) For any $\delta_{0}>0, u^{m}$ has a modulus of continuity $\omega_{e}(r)$ in $R^{n} \times\left[\delta_{0}, \infty\right)$ for any $0<\varepsilon<2 / n$, if $n \geqslant 3$, and a modulus of continuity $\tilde{\omega}(r)$ in $R^{n} \times\left[\delta_{0}, \infty\right)$ if $n=2$. 
The proof of continuity for $t>0$ and the proof of (ii) are given in \$4. It will become obvious from the proof that the condition (1.3) is not required for this part of the theorem.

The proof of continuity for $t=0$ is given in $\$ 5$.

$\$ \S 2$ and 3 develop some estimates needed in $\$ 4$.

2. Preliminary lemmas. In this section and in $\$ 3$ we obtain various auxiliary results for the solution $u_{\eta}(x, t)$ of (1.4), (1.8). For simplicity we shall denote this solution by $u(x, t)$; we also take $0<\eta<1$.

All the estimates which we shall obtain, and all the constants will be independent of $\eta$. We set $M=N+1$, so that, by (1.9),

$$
0<u(x, t)<M .
$$

LEMMA 2.1. The following inequalities hold:

$$
\begin{gathered}
t \frac{\partial u}{\partial t} \geqslant-\frac{u}{m-1}, \\
t \frac{\partial u^{m}}{\partial t} \geqslant-\frac{m}{m-1} u^{m} .
\end{gathered}
$$

This result is due to Aronson and Benilan [4]. Since the proof is short, we briefly give it here. The function $w=t(\partial u / \partial t)$ satisfies

$$
\partial w / \partial t=m \Delta\left(u^{m-1} w\right)+\Delta u^{m}
$$

The function $z=-u /(m-1)$ satisfies the same equation, and $z(x, 0)<0$ $=w(x, 0)$ ( $w$ is continuous at $t=0$ if $u_{0}(x)$ is smooth). By comparison, then, (2.2) follows if $u_{0}(x)$ is smooth; for general $u_{0}$, one uses approximation.

The inequality (2.3) follows immediately from (2.2).

Let $\delta_{0}$ be a fixed positive number. For any $x^{0} \in R^{n}, t^{0}>2 \delta_{0}$, we introduce the sets

$$
\begin{aligned}
C_{r, h}\left(x^{0}, t^{0}\right) & =\left\{(x, t) ;\left|x-x^{0}\right|<r, t^{0}-h<t<t^{0}\right\}, \\
B_{r}\left(x^{0}\right) & =\left\{x ;\left|x-x^{0}\right|<r\right\},
\end{aligned}
$$

where $h<\delta_{0}$. Denote the volume of $B_{r}\left(x^{0}\right)$ by $\left|B_{r}\left(x^{0}\right)\right|$. Set

$$
C_{0}=m /(m-1) \delta_{0}
$$

and let $h_{0}=h_{0}\left(\delta_{0}\right)$ be any positive number satisfying:

$$
e^{2 C_{0} h_{0}}<\frac{4}{3}, \quad h_{0}<\delta_{0} .
$$

LEMMA 2.2. For any $x^{0} \in R^{n}, t^{0}>2 \delta_{0}, \lambda>0, r>0,0<h<h_{0}\left(\delta_{0}\right)$, the following is true: if

$$
\frac{1}{\left|B_{r}\left(x^{0}\right)\right|} \int_{B_{r}\left(x^{0}\right)} u^{m}\left(x, t^{0}-h\right) d x \geqslant \lambda
$$

and if

$$
h>M \gamma_{n} r^{2} / \lambda,
$$


then

$$
u^{m}\left(x^{0}, t^{0}\right)>\frac{1}{2} \lambda
$$

here $\gamma_{n}$ is a positive number depending only on the dimension $n$.

Proof. By (2.3), (2.4), $\partial u^{m} / \partial t>-(m /(m-1) t) u^{m}>-C_{0} u^{m}$ if $t^{0}-h_{0}$ $<t<t^{0}$ (since $\left.t>2 \delta_{0}-h_{0}>\delta_{0}\right)$. Hence

$$
u^{m}\left(x^{0}, t^{0}\right)>e^{-C_{0}\left(t-t^{0}+h\right)} u^{m}\left(x^{0}, t\right)>e^{-C_{0} h_{0} u^{m}}\left(x^{0}, t\right)
$$

Again, by (2.3), (2.4), the function

$$
\varphi(t)=\frac{1}{\left|B_{r}\left(x^{0}\right)\right|} \int_{B_{r}\left(x^{0}\right)} u^{m}(x, t) d x
$$

satisfies

$$
\varphi^{\prime}(t)>-(m /(m-1) t) \varphi(t)>-C_{0} \varphi(t)
$$

so that

$$
\varphi(t)>e^{-C_{0}\left(t-t^{0}+h\right)} \varphi\left(t_{0}-h\right)>e^{-C_{0} h_{0}} \varphi\left(t_{0}-h\right)>e^{-C_{0} h_{0}} \lambda,
$$

where (2.6) was used in the last inequality.

Suppose $n>3$ and let

$$
G(\rho)=\rho^{2-n}-r^{2-n}-((n-2) / 2) r^{-n}\left(r^{2}-\rho^{2}\right), \quad \rho=\left|x-x^{0}\right| .
$$

Notice that $G(r)=0, G^{\prime}(r)=0$ Since $G^{\prime}(\rho)<0$ if $\rho<r, G(\rho)$ is positive in $B_{r}\left(x^{0}\right)$. By Green's formula,

$$
u^{m}\left(x^{0}, t\right)=\frac{1}{4} \gamma_{n} \int_{B_{r}\left(x^{0}\right)} G \Delta u^{m} d x+\frac{1}{\left|B_{r}\left(x^{0}\right)\right|} \int_{B_{r}\left(x^{0}\right)} u^{m}(x, t) d x
$$

where $\gamma_{n}$ is a positive constant depending only on $n$.

Suppose now that the assertion (2.8) is not true. Then (2.9) gives

$$
u^{m}\left(x^{0}, t\right)<\frac{1}{2} \lambda e^{c_{0} h_{0}} .
$$

Substituting this and (2.10) into (2.12) and using the first inequality of (2.5), we obtain

$$
\frac{1}{4} \lambda<\frac{1}{4} \gamma_{n} \int_{B_{r}\left(x^{0}\right)} G \Delta u^{m} d x
$$

Integrating this inequality with respect to $t, t^{0}-h<t<t^{0}$, we get

$$
\begin{aligned}
\lambda h & <\gamma_{n} \int_{t^{0}-h}^{t^{0}} \int_{B_{r}\left(x^{0}\right)} G(\rho) \Delta u^{m}(x, t) d x d t \\
& =\gamma_{n} \int_{t^{0}-h}^{t^{0}} \int_{B_{r}\left(x^{0}\right)} G(\rho) \frac{\partial u(x, t)}{\partial t} d x d t \\
& \leqslant \gamma_{n} \int_{B_{r}\left(x^{0}\right)} G(\rho) u\left(x, t^{0}\right) d x \leqslant \gamma_{n} M \int_{B_{r}\left(x^{0}\right)} G(\rho) d x .
\end{aligned}
$$


Since the right-hand side is bounded by $\gamma_{n} M r^{2}$, with another constant $\gamma_{n}$, we obtain a contradiction to (2.7). This completes the proof in case $n>3$. For $n=2$ the proof is the same provided we replace $G(\rho)$, defined in (2.11), by $G(\rho)=\log (r / \rho)-\frac{1}{2} r^{-2}\left(r^{2}-\rho^{2}\right)$.

In the next lemma we take $h_{0}=h_{0}\left(\delta_{0}\right)$ to satisfy, in addition to (2.5), the inequality

$$
h_{0}<2 n(m-1) \delta_{0} c / M, \quad c>0,
$$

and let $C_{1}$ be any constant satisfying:

$$
C_{1}-1>2^{n+1}, \quad C_{1}-1>\gamma_{n} M 2^{n+2} .
$$

LEMMA 2.3. Let $t^{0}>\delta_{0}, 0<h<h_{0}\left(\delta_{0}\right), \lambda=c \sigma(h)$ where $\sigma(h)>0, \sigma(h) \rightarrow 0$ if $h \rightarrow 0$. Let $x^{0}$ be any point in $R^{n}$ such that

$$
\left|x^{0}\right|<h^{1 / 2}(\sigma(h))^{1 / 2} \equiv r .
$$

If

$$
u^{m}\left(x^{0}, t^{0}\right)>C_{1} \lambda
$$

then

$$
u^{m}\left(0, t^{0}+h\right)>\lambda .
$$

Proof. Let $v(x)=u^{m}\left(x, t^{9}\right)+c_{1}\left|x-x^{0}\right|^{2}, c_{1}>0$. Then by Lemma 2.1 and (2.1),

$$
\Delta v=\partial u / \partial t+2 n c_{1}>-M /(m-1) \delta_{0}+2 n c_{1}=0
$$

provided

$$
c_{1}=M / 2 n(m-1) \delta_{0}
$$

Thus, $v$ is subharmonic. This implies that, for any $r>0$,

$$
\frac{1}{\left|B_{r}\left(x^{0}\right)\right|} \int_{B_{r}\left(x^{0}\right)} v>v\left(x^{0}\right)=u^{m}\left(x^{0}, t^{0}\right)>C_{1} \lambda,
$$

where (2.16) was used. Since the left-hand side is bounded above by

$$
c r^{2}+\frac{1}{\left|B_{r}\left(x^{0}\right)\right|} \int_{B_{r}\left(x^{0}\right)} u^{m},
$$

we obtain, if $r$ is taken as in (2.15),

$$
I \equiv \frac{1}{\left|B_{r}\left(x^{0}\right)\right|} \int_{B_{r}\left(x^{0}\right)} u^{m}>C_{1} \lambda-c_{1} r^{2}=C_{1} \lambda-c_{1} h \sigma(h) .
$$

Recalling the definition of $\lambda, c_{1}$ and the restriction (2.13), we find that $I>\left(C_{1}-1\right) \lambda$. If we now assume that $\left|x^{0}\right|<r$ then $B_{2 r}(0) \supset B_{r}\left(x^{0}\right)$, and we conclude that

$$
\frac{1}{\left|B_{2 r}(0)\right|} \int_{B_{2 r}(0)} u^{m}>\left(C_{1}-1\right) \lambda \frac{1}{2^{n}}
$$


We now wish to apply Lemma 2.2 with $\lambda, r$ replaced by $\lambda^{\prime}=\left(C_{1}-1\right) \lambda 2^{-n}$, $r^{\prime}=2 r$, and with $t^{0}-h$ replaced by $t^{0}$. The condition (2.7) is satisfied by virtue of the second inequality in (2.14). We conclude that $u^{m}\left(0, t^{0}+h\right)$ $>\frac{1}{2} \lambda^{\prime}>\lambda$, where the last inequality is a consequence of the first inequality in (2.14).

We conclude this section with the following lemma.

LEMMA 2.4. Let $\delta_{0}>0$ and let

$$
c_{*}=1 / m M^{m-1} \text {. }
$$

Then, for all $t>\delta_{0}$,

$$
\begin{gathered}
\Delta u^{m}>-M /(m-1) \delta_{0} \\
\Delta u^{m}-c_{*} \partial u^{m} / \partial t>-M /(m-1) \delta_{0} .
\end{gathered}
$$

Proof. By Lemma 2.1,

$$
\Delta u^{m}=\frac{\partial u}{\partial t}>-\frac{u}{(m-1) t}>-\frac{M}{(m-1) \delta_{0}},
$$

and (2.20) follows. To prove (2.21), we can write

$$
\begin{aligned}
\Delta u^{m}-c_{*} \frac{\partial u^{m}}{\partial t} & =\frac{\partial u}{\partial t}-c_{*} m u^{m-1} \frac{\partial u}{\partial t}=\left(1-c_{*} m u^{m-1}\right) \frac{\partial u}{\partial t} \\
& \geqslant-\left(1-c_{*} m u^{m-1}\right) \frac{u}{(m-1) t},
\end{aligned}
$$

since $1-c_{*} m u^{m-1}>0$ by (2.19). Since the right-hand side is $>-u /(m-$ $1) t>-M /(m-1) \delta_{0},(2.21)$ follows.

3. A priori estimates. The main result of this section is stated in Lemma 3.3.

Let $\varepsilon$ be any positive number such that $\varepsilon<2 / n$.

Fix a point $\left(x^{0}, t^{9}\right)$ in $R^{n} \times\left(2 \delta_{0}, \infty\right)$ and define

$$
R_{k}=\left\{(x, t):\left|x-x^{0}\right|<2^{-k}, t^{0}-2^{-2 k}<t<t^{0}\right\},
$$

where $k$ is a positive integer. In Lemma 3.1 we shall be interested only in $k>k^{*}$. Here $k^{*}$ is a positive number sufficiently large, to be determined in the proof of Lemma 3.1. It depends only on $\varepsilon, \delta_{0}, N$ and it also satisfies the inequality $2^{-2 k^{*}}<h_{0}$ where $h_{0}=h_{0}\left(\delta_{0}\right)$ is the number satisfying all the restrictions imposed in Lemmas 2.2, 2.3.

Define

$$
\mu_{k}=\sup _{R_{k}} u^{m}, \quad M_{k}=\max \left\{\mu_{k}, 4 C_{1} k^{-\varepsilon}\right\},
$$

where $C_{1}$ is the constant appearing in Lemma 2.3 .

LEMMA 3.1. Suppose

$$
u^{m}\left(x^{0}, t^{0}\right)<k_{0}^{-\varepsilon}
$$


for some $k_{0}>k^{*}$. Then, for any $k^{*}<k<k_{0}$,

$$
\mu_{k+1} \leqslant M_{k}\left(1-C k^{-e n / 2}\right)
$$

where $C$ is a constant depending only on $\varepsilon, \delta_{0}, N$.

Proof. We wish to apply Lemma 2.3 with $\sigma(h)=|\log h|^{-e}, h=2^{-2 k}$, $c=(2 \log 2)^{e}$ (so that $\left.\lambda=k^{-\varepsilon}\right)$. The lemma asserts that if $u^{m}\left(x, t^{0}-2^{-2 k}\right)>$ $C_{1} \lambda$ for some $x$ such that $\left|x-x^{0}\right|<2^{-k} k^{-\varepsilon / 2}$, then $u\left(x^{0}, t^{0}\right)>\lambda$. Since the last inequality contradicts (3.1) (since $k<k_{0}$ ), it follows that

$$
u^{m}\left(x, t^{0}-2^{-2 k}\right)<C_{1} k^{-\varepsilon} \text { if }\left|x-x^{0}\right|<2^{-k} k^{-e / 2}
$$

for all $k^{*}<k<k_{0}$.

Let

$$
v(x)=u^{m}\left(x, t^{0}-h\right)+c_{1}\left|x-x^{*}\right|^{2} \quad \text { where }\left|x^{*}-x^{0}\right|<\frac{1}{3} 2^{-k} ;
$$

$c_{1}$ is chosen as in (2.18), so that $\Delta v>0$.

Denote by $B$ the ball with center $x^{*}$ and radius $\frac{2}{3} 2^{-k}$, and denote by $B^{\prime}$ the ball with center $x^{0}$ and radius $2^{-k} k^{-\varepsilon / 2}$. Then $B^{\prime}$ is contained in $B$ (if $\left.k>k^{*}\right)$.

Since $v$ is subharmonic,

$$
\begin{aligned}
u^{m}\left(x^{*}, t^{0}-h\right) & =v\left(x^{*}, t^{0}-h\right)<\frac{1}{|B|} \int_{B} v(x) d x \\
& <c_{1}\left(\frac{2}{3} 2^{-k}\right)^{2}+\frac{1}{|B|} \int_{B} u^{m}\left(x, t^{0}-h\right) d x .
\end{aligned}
$$

Using the inequality (3.3) in $B^{\prime}$, and the inequality $u^{m}\left(x, t^{0}-h\right)<M_{k}$ in $B-B^{\prime}$, we can estimate the last term in (3.5) by $M_{k}\left(1-k^{-e n / 2}\right)+$ $C_{1} k^{-e n / 2} k^{-e}$. Substituting this estimate in (3.5) we get

$$
u^{m}\left(x^{*}, t^{0}-h\right)<M_{k}\left(1-k^{-e n / 2}\right)+2 C_{1} k^{-e n / 2} k^{-\varepsilon} \text {. }
$$

Recalling the $M_{k}>4 C_{1} k^{-\varepsilon}$, we conclude (if $k>k^{*}$ ) that

$$
u^{m}\left(x^{*}, t^{0}-h\right)<M_{k}\left(1-\frac{1}{2} k^{-e n / 2}\right) \text { if }\left|x^{*}-x^{0}\right|<\frac{1}{3} 2^{-k} \text {. }
$$

Let $x^{\prime}=\left(x-x^{9}\right) / 2^{k}, t^{\prime}=\left(t-t^{9}\right) / 2^{2 k}, w\left(x^{\prime}, t^{\prime}\right)=u^{m}(x, t)$.

By Lemma 2.4,

$$
\left(\Delta-c_{*} \partial / \partial t^{\prime}\right) w=2^{-2 k}\left(\Delta-c_{*} \partial / \partial t\right) u^{m}>-\tilde{C} 2^{-2 k}
$$

where $\tilde{C}=M /\left(\delta_{0}(m-1)\right)$.

Consider the function

$$
z=1-w / M_{k}
$$

in the cylinder $\left|x^{\prime}\right|<1,-1<t^{\prime}<0$. Clearly $z>0$ in this cylinder. Also, by (3.6),

$$
z\left(x^{\prime},-1\right)>\frac{1}{2} k^{-e n / 2} \text { if }\left|x^{\prime}\right|<\frac{1}{3}
$$


By (3.7) we further have

$$
\left(\Delta-c_{*} \partial / \partial t^{\prime}\right) z<\tilde{C} 2^{-2 k} / M_{k} .
$$

Denote by $G\left(x^{\prime}, \xi^{\prime}, t^{\prime}\right)$ the Green function of $\Delta-c_{*}\left(\partial / \partial t^{\prime}\right)$ in the cylinder $\left|x^{\prime}\right|<1,-1<t^{\prime}<0$. Representing $z$ in terms of Green's function, and using (3.9), (3.10), we get

$$
\begin{aligned}
z\left(x^{\prime}, t^{\prime}\right) \geqslant & -\int_{-1}^{t^{\prime}} \int_{\left|\xi^{\prime}\right|<1} \frac{\tilde{C} 2^{-2 k}}{M_{k}} G\left(x^{\prime}, \xi^{\prime}, t^{\prime}-s\right) d \xi^{\prime} d s \\
& +\frac{1}{2} k^{-e n / 2} \int_{\left|\xi^{\prime}\right|<1 / 3} G\left(x^{\prime}, \xi^{\prime}, t^{\prime}+1\right) d \xi^{\prime}
\end{aligned}
$$

We restrict $\left(x^{\prime}, t^{\prime}\right)$ to the set $\left|x^{\prime}\right|<\frac{1}{2},-\frac{1}{2}<t^{\prime}<0$. Then the last integral is larger than a positive constant $4 c$, and

$$
\int_{-1}^{t^{\prime}} \int_{\left|\xi^{\prime}\right|<1} G\left(x^{\prime}, \xi^{\prime}, s\right) d \xi^{\prime} d s<C,
$$

where $c$ and $C$ depend only on $c_{*}, n$. It follows that

$$
z\left(x^{\prime}, t^{\prime}\right)>2 c k^{-e n / 2}-C \tilde{C} 2^{-2 k} / M_{k}>c k^{-e n / 2},
$$

where the inequality $M_{k} \geqslant 4 C_{1} k^{-e}$ was used; here again we take $k>k^{*}$ with $k^{*}$ sufficiently large.

Recalling now (3.8), the assertion (3.2) follows from (3.12).

LEMMA 3.2. Under the assumptions of Lemma 3.1,

$$
\mu_{k}<\hat{C} k^{-\varepsilon} \text { for all } k<k_{0}
$$

where $\hat{C}$ is a constant depending only on $\varepsilon, \delta_{0}, N$.

Proof. We choose $\hat{C}$ so large that (3.13) holds for all $1<k<k^{*}+1$ and $\hat{C}>1$. Next we proceed by induction on $k$. Suppose (3.13) holds for some $k$, $k^{*}<k<k_{0}$; we shall prove it for $k+1$. In view of the definition of $M_{k}$, $M_{k}<\hat{C} k^{-e}$. Substituting this into (3.2) we get

$$
\mu_{k+1}<\hat{C} k^{-e}\left(1-C k^{-e n / 2}\right)<\hat{C}(k+1)^{-\varepsilon}
$$

provided

$$
1-\frac{C}{k^{e n / 2}}<\left(\frac{k+1}{k}\right)^{-e},
$$

which is certainly the case if $k^{*}$ is sufficiently large (depending on $\varepsilon, C$ ) since $\mathrm{en} / 2<1$.

LEMMA 3.3. For any $0<\varepsilon<2 / n, \delta_{0}>0$ there exists a constant $C^{*}>1+$ $N$, depending only on $\varepsilon, \delta_{0}, N$, such that for any $(x, t),\left(x^{0}, t^{0}\right)$ in $R^{n} \times\left(\delta_{0}, \infty\right)$,

$$
u^{m}(x, t)<C^{*} \max \left\{\left|\log \left(\left|x-x^{0}\right|+\left|t-t^{0}\right|^{1 / 2}\right)\right|^{-\varepsilon}, u^{m}\left(x^{0}, t^{0}\right)\right\}
$$


Proof. We may assume that $u^{m}\left(x^{0}, t^{0}\right)<1$.

Let $k_{0}$ be a positive integer such that

$$
\left(k_{0}+1\right)^{-e}<u^{m}\left(x^{0}, t^{0}\right) \leqslant\left(k_{0}\right)^{-e} .
$$

Consider the first case where $k_{0}>k^{*}$. By Lemma 3.2,

$$
u^{m}(x, t)<\hat{C} k^{-e} \text { if }\left|x-x^{0}\right|<2^{-k}, t^{0}-2^{-2 k}<t<t^{0},
$$

provided $k<k_{0}$. This gives

$$
u^{m}(x, t)<C\left|\log \left(\left|x-x^{0}\right|+\left|t-t^{0}\right|^{1 / 2}\right)\right|^{-e}
$$

as long as $(x, t)$ does not satisfy: $\left|x-x^{0}\right|<2^{-k_{0}}, t^{0}-2^{-2 k_{0}}<t<t^{0}$. If, on the other hand $\left|x-x^{0}\right|<2^{-k_{0}}, t^{0}-2^{-2 k_{0}}<t<t^{0}$, then (3.16), with $k=k^{0}$, gives

$$
u^{m}(x, t)<\hat{C}\left(k_{0}\right)^{-\varepsilon}<C u^{m}\left(x^{0}, t^{0}\right),
$$

where (3.15) has been used. Thus we have proved (3.14) if $t<t^{0}$ and $k_{0}>k^{*}$. If $k_{0}<k^{*}$ then $u^{m}\left(x^{0}, t^{9}\right)>\left(k^{*}+1\right)^{-\varepsilon}$ and (3.14) follows by choosing $C^{*}>M\left(k^{*}+1\right)^{e}$. We have thus completed the proof of $(3.14)$ in case $t<t^{0}$. In particular it follows that

$$
u^{m}\left(x, t^{0}\right)<C^{*}|\log | x-x^{0}||^{-\varepsilon}+C^{*} u^{m}\left(x^{0}, t^{0}\right) .
$$

Let $v$ be the solution of

$$
\begin{gathered}
\Delta v-c_{*} \frac{\partial v}{\partial t}=-\frac{M}{2(m-1) \delta_{0}} \quad \text { in } R^{n} \times\left(t^{0}, \infty\right), \\
v\left(x, t^{0}\right)=u^{m}\left(x, t^{0}\right) \quad \text { in } R^{n} .
\end{gathered}
$$

Since $u^{m}$ satisfies (2.21), we can compare $v$ with $u^{m}$ and conclude that

$$
u^{m}(x, t)<v(x, t) \text {. }
$$

Representing $v$ in terms of the fundamental solution of $\Delta-c_{*}(\partial / \partial t)$ and then using (3.17), we find that

$$
v(x, t)<C^{*}\left|\log \left(\left|x-x^{0}\right|+\left|t-t^{0}\right|^{1 / 2}\right)\right|^{-\varepsilon}+C^{*} u^{m}\left(x^{0}, t^{0}\right) \text { if } t>t^{0},
$$

with another constant $C^{*}$. Using (3.18), the inequality (3.14) then follows (with yet another constant $C^{*}$ ) for $t>t^{0}$.

4. Proof of Theorem 1.1 for $t>0$. We begin by deriving another version of Lemma 3.3.

LemMA 4.1. For any $0<\varepsilon<2 / n, \delta_{0}>0$, if $\left(x^{i}, t^{i}\right) \in R^{n} \times\left(2 \delta_{0}, \infty\right)$ for $i=0,1$ and if

$$
\left|\log \left(\left|x^{1}-x^{0}\right|+\left|t^{1}-t^{0}\right|^{1 / 2}\right)\right|^{-e}<\left(1 / C^{*}\right) u^{m}\left(x^{i}, t^{i}\right)
$$

for $i=0$ or $i=1$, then

$$
1 / C^{*}<u^{m}\left(x^{1}, t^{1}\right) / u^{m}\left(x^{0}, t^{0}\right)<C^{*} .
$$

Here $C^{*}$ is the same constant as in Lemma 3.3. 
Proof. It suffices to prove (4.2) when (4.1) holds for $i=0$, that is,

$$
\left|\log \left(\left|x^{1}-x^{0}\right|+\left|t^{\prime}-t^{0}\right|^{1 / 2}\right)\right|^{-e}<\left(1 / C^{*}\right) u^{m}\left(x^{0}, t^{0}\right) \text {. }
$$

The inequality $u^{m}\left(x^{1}, t^{1}\right)<C^{*} u^{m}\left(x^{0}, t^{9}\right)$ is of course a consequence of (3.14) and (4.1) (for $i=0)$. To prove that $u^{m}\left(x^{1}, t^{1}\right)>u^{m}\left(x^{0}, t^{0}\right) / C^{*}$, we proceed by assuming that

$$
u^{m}\left(x^{1}, t^{1}\right)<\left(1 / C^{*}\right) u\left(x^{0}, t^{0}\right)
$$

and deriving a contradiction.

We write (3.14) with $(x, t)$ and $\left(x^{0}, t^{0}\right)$ replaced, respectively, by $\left(x^{0}, t^{9}\right)$ and $\left(x^{1}, t^{1}\right)$ and then use the relations (4.3), (4.4). We obtain $u^{m}\left(x^{0}, t^{0}\right)<$ $u^{m}\left(x^{0}, t^{0}\right)$, which is impossible.

Take now any point $\left(x^{0}, t^{0}\right)$ with $t_{0}>2 \delta_{0}$, and let $k_{0}$ be a positive integer such that

$$
M^{m}\left(k_{0}+1\right)^{-\varepsilon}<u^{m}\left(x^{0}, t^{0}\right)<M^{m} k_{0}^{-\varepsilon} .
$$

Define

$$
\Sigma_{0}=\left\{(x, t):\left|x-x^{0}\right|+\left|t-t^{0}\right|^{1 / 2}<2^{-\mu c k_{0}}, t>\delta_{0}\right\}
$$

where $c$ and $\mu$ are positive numbers to be determined below (independently of $\left.k_{0}\right)$, and $\mu>2$.

If $(x, t) \notin \Sigma_{0}, t>\delta_{0}$, then $\left|\log \left(\left|x-x^{0}\right|+\left|t-t^{0}\right|^{1 / 2}\right)\right|^{-e}>\left(k_{0} \mu c \log 2\right)^{-e}$. Recalling (4.5) and using Lemma 3.3, we then obtain $u^{m}(x, t)<C \mid \log (\mid x-$ $\left.x^{0}|+| t-\left.t^{0}\right|^{1 / 2}\right)\left.\right|^{-e}$ where $C$ is a positive constant depending on $\delta_{0}, \varepsilon, N, \mu, c$. Since the same inequality holds also for $u^{m}\left(x^{0}, t^{0}\right)$, we obtain

$$
\left|u^{m}(x, t)-u^{m}\left(x^{0}, t^{0}\right)\right|<2 C\left|\log \left(\left|x-x^{0}\right|+\left|t-t^{0}\right|^{1 / 2}\right)\right|^{-e} \text {. }
$$

We shall now evaluate the left-hand side for $(x, t) \in \Sigma_{0}$. By (4.5) and Lemma 4.1,

$$
1 / C^{*}<u^{m}(\bar{x}, \bar{t}) / u^{m}\left(x^{0}, t^{0}\right)<C^{*}
$$

provided

$$
\left|\log \left(\left|\bar{x}-x^{0}\right|+\left|\bar{t}-t^{0}\right|^{1 / 2}\right)\right|^{-\varepsilon}<\left(N^{m} / C^{*}\right)\left(k_{0}+1\right)^{-\varepsilon}
$$

that is, provided $\left|\bar{x}-x^{0}\right|+\left|\bar{t}-t^{0}\right|^{1 / 2}<2^{-\bar{c} k_{0}}$ where $\bar{c}$ is a positive constant depending on $\varepsilon, \delta_{0}, N$. We now choose, in (4.6), $c=\bar{c}$. It follows that if

$$
\left|\bar{x}-x^{0}\right|<2^{-c k_{0}}, \quad\left|\bar{t}-t^{0}\right|<2^{-2 c k_{0}}
$$

then (4.8) holds.

Introduce variables

$$
x^{\prime}=\left(x-x^{0}\right) / 2^{-c k_{0}} \lambda, \quad t^{\prime}=\left(t-t^{0}\right) / 2^{-2 c k_{0}},
$$

where $\lambda=k_{0}^{-(m-1) e / 2 m}$ and let $v\left(x^{\prime}, t^{\prime}\right)=u(x, t)$. Then

where $a=m u^{m-1} k_{0}^{e(m-1) / m}$.

$$
\partial v / \partial t^{\prime}=\nabla\left(a\left(x^{\prime}, t^{\prime}\right) \nabla v^{\prime}\right)
$$


If

$$
\left|x^{\prime}\right| \leqslant k_{0}^{e(m-1) / m}, \quad\left|t^{\prime}\right|<1,
$$

then

$$
\left|x-x^{0}\right| \leqslant 2^{-c k_{0}} \lambda^{2} k_{0}^{e(m-1) / m}=2^{-c k_{0}}, \quad\left|t-t^{0}\right|<2^{-2 c k_{0}},
$$

so that (4.9) holds with $(\bar{x}, \bar{t})=(x, t)$; consequently also (4.8) is thus satisfied. Then, $C_{1}<a\left(x^{\prime}, t^{\prime}\right)<C_{2}$ where $C_{1}, C_{2}$ are positive constants depending only on $\varepsilon, \delta_{0}, N$. We can now apply the Nash estimate [8] to $v$ and conclude that for some $\alpha, 0<\alpha<1$,

$$
\begin{aligned}
\left|u(x, t)-u\left(x^{0}, t^{0}\right)\right| & =\left|v\left(x^{\prime}, t^{\prime}\right)-v(0,0)\right|<C\left(\left|x^{\prime}\right|^{\alpha}+\left|t^{\prime}\right|^{\alpha / 2}\right) \\
& =C \frac{\left|x-x^{0}\right|^{\alpha}}{\left(2^{-c k_{0}} \lambda\right)^{\alpha}}+C \frac{\left|t-t^{0}\right|^{\alpha / 2}}{\left(2^{-2 c k_{0}}\right)^{\alpha}}
\end{aligned}
$$

where $C$ is a generic constant depending only on $\varepsilon, \delta_{0}, N$.

Now, if we take $\mu>4 / \alpha$ then, for $(x, t) \in \Sigma_{0}$,

$$
\begin{gathered}
\left|x-x^{0}\right|^{\alpha / 2}<\left(2^{-\mu c k_{0}}\right)^{\alpha / 2}<\left(2^{-c k_{0}} \lambda\right)^{\alpha}, \\
\left|t-t^{0}\right|^{\alpha / 4}<\left(2^{-2 \mu c k_{0}}\right)^{\alpha / 4}<\left(2^{-2 c k_{0}}\right)^{\alpha} .
\end{gathered}
$$

Substituting this into (4.12), we get

$$
\left|u(x, t)-u\left(x^{0}, t^{0}\right)\right|<C\left(\left|x-x^{0}\right|^{\alpha / 2}+\left|t-t^{0}\right|^{\alpha / 4}\right) .
$$

Combining this with (4.7) we find that (4.7) (with a different $C$ ) is valid for all $(x, t),\left(x^{0}, t^{0}\right)$ in $R^{n} \times\left(2 \delta_{0}, \infty\right)$.

We now recall that the function $u$ which we have been considering so far is actually the solution $u_{\eta}$ of (1.4), (1.8), and $u_{\eta}(x, t) \downarrow u(x, t)$ as $\eta \downarrow 0$. Hence, by taking $\eta \downarrow 0$ in (4.7) (for $u_{\eta}$ ) we obtain the same inequality for $u$. This completes the proof of Theorem 1.1(ii) in case $n>3$.

If $n=2$ we can improve the modulus of continuity. We take in the proof of Lemma $3.1 \sigma(h)=2^{-|\log h|^{1 / 2}}, h=2^{-2 k}, \lambda=2^{-k^{1 / 2}}$ and apply Lemma 2.3 (with $r=2^{-k} 2^{-k^{1 / 2}}$ ). The function $z$ defined in (3.8) then satisfies (3.10) and

$$
z\left(x^{\prime},-1\right)>1-c 2^{-k^{1 / 2}} / \mu_{k}>\frac{1}{2} \text { if }\left|x^{\prime}\right|<2^{-k^{1 / 2}},
$$

where $M_{k}=\max \left\{\mu_{k}, 2^{-C^{\prime} k^{1 / 2}}\right\}$ and $c, C^{\prime}$ are positive constants. Let $\zeta\left(x^{\prime}\right)=$ $z\left(x^{\prime},-1\right)-\left(C 2^{-2 k} / M_{k}\right)\left|x^{\prime}\right|^{2}$. Then $\left(\Delta-c_{*} \partial / \partial t^{\prime}\right) \zeta<0$. Also

$$
\begin{aligned}
& \zeta>\frac{1}{3} \quad \text { if }\left|x^{\prime}\right|<2^{-k^{1 / 2}}, \\
& \zeta \geqslant-C 2^{-2 k} / M_{k} \text { if }\left|x^{\prime}\right|=1 .
\end{aligned}
$$

We compare $\zeta$ with the function

$$
\eta\left(x^{\prime}\right)=\frac{1}{4} \frac{\log \left|x^{\prime}\right|}{\log 2^{-k^{1 / 2}}}-\frac{C 2^{-2 k}}{M_{k}} .
$$


By the maximum principle, $\zeta>\eta$ if $2^{-k^{1 / 2}}<\left|x^{\prime}\right|<1$. Hence, if $\left|x^{\prime}\right|<\frac{1}{2}$,

$$
\begin{aligned}
z\left(x^{\prime},-1\right) & >\frac{1}{2} \frac{\log (1 / 2)}{\log 2^{-k^{1 / 2}}}-\frac{C 2^{-2 k}}{M_{k}}+\frac{C 2^{-2 k}}{M_{k}}\left|x^{\prime}\right|^{2} \\
& >\frac{C}{k^{1 / 2}}-C 2^{-k}>\frac{C}{k^{1 / 2}},
\end{aligned}
$$

where $C$ is a generic constant depending on $\delta_{0}, N$.

We can now proceed as in (3.11) and obtain

$$
z\left(x^{\prime}, t^{\prime}\right)>C / k^{1 / 2} \quad \text { if }\left|x^{\prime}\right|<\frac{1}{2},-\frac{1}{2}<t^{\prime}<0 .
$$

Therefore $\mu_{k+1}<M_{k}\left(1-C / k^{1 / 2}\right)$ for any $k>k^{*}$.

Proceeding analogously to the proof of Lemma 3.2, we establish by induction on $k$ that

$$
\mu_{k}<C 2^{-C^{\prime} k^{1 / 2}} \quad\left(k^{*}<k<k_{0}\right) .
$$

provided $C^{\prime}$ is chosen sufficiently small.

With (4.13) at hand, we can now proceed as in the case $n>3$, replacing everywhere the modulus of continuity $C|\log r|^{-e}$ by $C 2^{-C^{\prime}|\log r|^{1 / 2}}$.

5. Continuity at $t=0$. We first prove continuity at a point $(y, 0)$ where $u_{0}(y)>0$. Consider the function

$$
w(x, t)=\frac{1}{A t+1}\left(1-\frac{|x|^{2}}{(A t+1)^{\alpha}}\right)^{1 /(m-1)} \quad(\alpha>0, A>0) .
$$

By direct calculation we find that in the region where $|x|^{2}<A t+1,0<t<$ $\delta: \Delta w^{m}-w_{t}>0$ provided $B \equiv A^{m+\alpha-1}$ and $\alpha$ satisfy $B>2 m n /(m-1)$, $\alpha B<4 m /(m-1)$, and provided $\delta$ is sufficiently small. Define

$$
w_{c, L}(x, t)=c w\left(L x, c^{m-1} L^{2} t\right), \quad c>0, L>0 .
$$

Then (cf. [6])

$$
\Delta\left(w_{c, L}\right)^{m}-\partial w_{c, L} / \partial t>0 \text { if }|x|^{2}<A c^{m-1} t+L^{-2}, t<\delta_{0}
$$

where $\delta_{0}=\delta /\left(c^{m-1} L^{2}\right)$.

Consider the function

$$
v(x, t)= \begin{cases}w_{c, L}(x, t) & \text { if }|x|^{2}<A c^{m-1} t+L^{-2} \\ 0 & \text { if }|x|^{2}>A c^{m-1} t+L^{-2}\end{cases}
$$

Since $v^{m}$ vanishes on $|x|^{2}=A c^{m-1} t+L^{-2}$ to an order larger than 1 , it is clear that $v$ is a subsolution of (1.4). Further,

$$
\begin{array}{ll}
v(x, 0)=0 & \text { if }|x|>1 / L, \\
v(x, 0)<c & \text { if }|x|<1 / L .
\end{array}
$$


Now, since $u_{0}(y)>0$, we have $u_{0}(x)>c$ if $|x-y|<1 / L$ for some $c>0$, $L>0$. We can therefore compare the solution $u_{\eta}(x, t)$ with $\tilde{v}(x, t) \equiv v(x-$ $y, t)$, and conclude that

$$
u_{\eta}(x, t) \geqslant \tilde{v}(x, t) \text { if } 0<t<\delta_{0} .
$$

Consequently $u_{\eta}(x, t)>c / 2$ if $|x-y|<1 / 2 L, 0<t<\delta^{\prime} \quad\left(\delta^{\prime}\right.$ small enough).

We can now apply the Nash estimate [8] and deduce a uniform Hölder continuity on the $u_{\eta}(x, t)$ for

$$
|x-y|<1 / 3 L, \quad 0<t<\frac{1}{2} \delta^{\prime},
$$

with exponent and coefficient which are independent of $\eta$. Taking $\eta \rightarrow 0$, we conclude that $u(x, t)$ is also Hölder continuous in the set (5.6).

It remains to prove continuity at a point $(y, 0)$ for which $u_{0}(y)=0$. For any $\varepsilon>0$ there is a $\delta>0$ such that

$$
u_{0}(x)<\varepsilon \quad \text { if }|x-y|<\delta .
$$

Consider the parabolic problem

$$
\begin{aligned}
\partial w / \partial t & =\Delta w^{m} \quad \text { if }|x-y|<\delta, t>0, \\
w(x, 0) & =2 \varepsilon \quad \text { if }|x-y|<\delta, \\
w(x, t) & =N+\varepsilon \quad \text { if }|x-y|=\delta, t>0 .
\end{aligned}
$$

This problem has a classical solution. We shall compare this solution with the function $u_{\eta}(x, t)$ for $\eta<\varepsilon$. In view of (5.7),

$$
u_{\eta}(x, 0)<w(x, 0) \text { if }|x-y|<\delta .
$$

Since also $u_{\eta}(x, t)<w(x, t)$ when $|x-y|=\delta, t>0$, we conclude that $u_{\eta}(x, t)<w(x, t)$. Taking $\eta \rightarrow 0$ and noting that $w(x, t)$ is continuous at $(y, 0)$, we get

$$
\varlimsup_{(x, t) \rightarrow(y, 0)} u(x, t)<\lim _{(x, t) \rightarrow(y, 0)} w(x, t)=2 \varepsilon .
$$

Since $\varepsilon$ is arbitrary, $u(x, t) \rightarrow 0=u_{0}(y)$ if $(x, t) \rightarrow(y, 0)$. This completes the proof.

Remark 1. Consider the case $n=1$. Then $u_{x x}^{m}>-c$ and, since $u^{m}$ is bounded, $u_{x}^{m}$ must then be bounded. Using the relation $u_{t}^{m}>-c u^{m}$ we then deduce that if $t_{2}>t$ then

$$
u^{m}\left(x_{2}, t_{2}\right)>u^{m}\left(x_{1}, t_{1}\right)-C\left(\left|x_{2}-x_{1}\right|+\left|t_{2}-t_{1}\right|\right) .
$$

If $v_{x x}-c_{*} v_{t}=-C\left(c_{*}>0, C>0\right)$ and $v\left(x, t_{1}\right)=u^{m}\left(x, t_{1}\right)$, then we get, upon using (5.8) with $t_{2}=t_{1}$, that

$$
\left|v(x, t)-v\left(x_{1}, t_{1}\right)\right|<C\left(\left|x-x_{1}\right|^{\theta}+\left|t-t_{1}\right|^{\theta / 2}\right) \quad\left(t>t_{1}\right)
$$

for any $\theta<\frac{1}{2}$. Since $u_{x x}^{m}-c_{*} u_{t}^{m}>-C$ for some $c_{*}>0, C>0$, we conclude that $u^{m}<v$, so that 


$$
u^{m}\left(x_{2}, t_{2}\right)<u^{m}\left(x_{1}, t_{1}\right)+C\left(\left|x_{2}-x_{1}\right|^{\theta}+\left|t_{2}-t_{1}\right|^{\theta / 2}\right) .
$$

Together with (5.8) we thus obtain a modulus of continuity $A r^{\theta}$ for $u^{m}$, for any $\theta<1$. Actually, in this case of $n=1$, a better modulus of continuity is known (Aronson [1], Gilding [5]): $\left|\left(u^{m-1}\right)_{x}\right|<C$, and

$$
\left|u\left(x_{2}, t_{2}\right)-u\left(x_{1}, t_{1}\right)\right|<C\left(\left|x_{2}-x_{1}\right|^{\nu}+\left|t_{2}-t_{1}\right|^{\nu / 2}\right)
$$

where $\nu=\min (1,1 /(m-1))$.

REMARK 2. Theorem 1.1 implies that the sets

$$
\begin{aligned}
\Omega & =\left\{(x, t) \in R^{n} \times(0, \infty) ; u(x, t)>0\right\}, \\
\Omega(t) & =\left\{x \in R^{n} ; u(x, t)>0\right\}
\end{aligned}
$$

are open subsets of $R^{n} \times(0, \infty)$ and $R^{n}$, respectively. The relation (2.9) (for $\left.u_{\eta}, \eta \rightarrow 0\right)$ implies

$$
u^{m}(x, t)>C\left(t, t_{0}\right) u^{m}\left(x, t_{0}\right)
$$

where $C\left(t, t_{0}\right)>0$. It easily follows that

$$
\Omega(t) \text { is increasing with } t \text {; }
$$

it is not necessarily strictly increasing (see [2], [3], [7]).

REMARK 3. The results of this paper extend to the more general equation

$$
u_{t}=\Delta \varphi(u)
$$

where $\varphi(0)=0, \varphi^{\prime}(0)=0, \varphi^{\prime}(u)>0$. The analog of Lemma 1.1, for this case, is proved in [4].

\section{REFERENCES}

1. D. G. Aronson, Regularity properties of flows through porous media, SIAM J. Appl. Math. 17 (1969), 461-467.

2. Appl. Math. 19 (1970), 299-307.

3. Mech. Anal. 37 (1970), 1-10.

4. D. G. Aronson and P. Benilan, Régularité des solutions de l'équation des milieux porous dans $R^{N}$, C. R. Acad. Sci. Paris, 1979.

5. B. H. Gilding, Continuity of generalized solutions of the Cauchy problem for the porous medium equation, J. London Math. Soc. 13 (1976), 103-106.

6. S. Kamenomostkaja, The asymptotic behavior of the solution of the filtration equation, Israel J. Math. 14 (1973), 76-87.

7. B. F. Knerr, The porous medium equation in one dimension, Trans. Amer. Math. Soc. 234 (1977), 381-415.

8. O. A. Ladyzhenskaja, V. A. Solonnikov and N. N. Ural'ceva, Linear and quasilinear equations of parabolic type, Trans. Math. Monographs, vol. 23, Amer. Math. Soc., Providence, R. I., 1968. 
9. O. A. Oleinik, On some degenerate quasilinear parabolic equations, Seminari dell' Istituto Nazionale di Alta Matematica 1962-1963, Oderisi, Gubbio, 1964, pp. 355-371.

10. O. A. Oleinik, A. S. Kalashnikov and C. Yui-Lin, The Cauchy problem and boundary problems for equations of the type of nonstationary filtration, Izv. Akad. Nauk SSSR Ser. Math. 22 (1958), 667-704.

11. E. S. Sabinina, On the Cauchy problem for the equation of nonstationary gas filtration in several space variables, Dokl. Akad. Nauk SSSR 136 (1961), 1034-1037.

Department of Mathematics, University of Minnesota, Minneapolis, Mnnnesota 55455

Department of Mathematics, Northwestern University, Evanston, ILLNois 60201 